\title{
Disminuyendo la Brecha del Aseguramiento de la Calidad entre Gestor y Miembros del Equipo Mediante el Uso de Herramientas de Software
}

\author{
Mirna Muñoz \\ mirna.munoz@cimat.mx \\ Centro de Investigación en Matemáticas- Unidad Zacatecas, Parque Quantum, Ciudad el Conocimiento \\ Avenida Lassec ,Andador Galileo Galilei, Manzana, 3 Lote 7, 9816o. Zacatecas, México.
}

DOI: 10.17013/risti.31.18-32

\begin{abstract}
Resumen: El desarrollo del software se ha vuelto cada vez más complejo, involucrando la coordinación de diversos profesionales involucrados en su desarrollo en distintos niveles jerárquicos como: gerentes de calidad, líderes de proyecto, desarrolladores, arquitectos de software, analistas de software y testers. Esta interacción lleva consigo el surgimiento de retos y/o limitantes que deben ser gestionados durante el desarrollo del proyecto. Desafortunadamente, la interacción y entendimiento de los objetivos de calidad establecidos no siempre son los esperados afectando la obtención de resultados esperados. Este artículo presenta los resultados de la validación de una herramienta desarrollada como apoyo para llevar a cabo actividades relacionadas con el aseguramiento de la calidad. Los resultados demuestran que la herramienta permite un entendimiento común entre los diferentes profesionales involucrados en el desarrollo de software referente a los objetivos de calidad establecidos, reforzando la comunicación y el análisis de resultados para toma de decisiones oportuna.
\end{abstract}

Palabras-clave: aseguramiento de la calidad; desarrollo de software; herramientas de software; calidad en el software.

\section{Reducing the Quality Assurance Gap between Middle Management and Operational through the Use of Software Tools}

Abstract: The development of software has become more complex, involving
the coordination of different processionals throughout its development, such
as: quality manager, project leader, developers, software architects, software
analyst, and testers. This interaction brings challenges and constraints that must
be managed during the development of software. Unfortunately, the interaction
and understanding of the quality goals established are not always the expected.
This paper presents the results of validate a tool developed as support to carry
out activities related to quality assurance. The results show that the tool allows a
common understanding among different professionals involved in the software
development regarding the quality goals established, reinforcing communication 
and the analysis of the quality assurance activities results analysis for a timely decision making.

Keywords: quality assurance; software development; software tools; software quality.

\section{Introducción}

Hoy en día asegurar la calidad del software se ha convertido es una necesidad debido a la incursión del software en diferentes sectores industriales como minería, automotriz, aeroespacial, agroindustria, turismo, etc., donde se está convirtiendo núcleo para la realización de sus actividades cotidianas (Muñoz, Mejia \& Ibarra, 2018). Además, 4 de 5 de las empresas más capitalizadas en el mundo bursátil son empresas de desarrollo de software (Rebolledo, 2017).

El desarrollo de software para estos distintos sectores industriales hace que su desarrollo se vuelva más complejo, involucrando la interacción entre profesionales de diversos niveles jerárquicos, dando como resultado el surgimiento tanto de retos y limitantes para lograr la calidad esperada (Suali, Fauzi, Sobri, \& Nasir, 2017).

En este contexto es importante distinguir el concepto de calidad del de aseguramiento de calidad (Muñoz, Mejía \& Ibarra, 2018): (1) calidad es el nivel en el que el producto de software cumple con los requisitos; (2) aseguramiento de la calidad son aquellas actividades que se realizan para generar evidencia que permite evaluar que los procesos y productos de software cumplen con los estándares aplicables, adecuados al tipo de producto de software que se desea obtener.

Por lo tanto, para que pueda ser lograda la calidad esperada en el producto se software, se deben ejecutar actividades que permitan evaluar, controla y tomar decisiones oportunas de acuerdo a los resultados. Esta situación resalta, por un lado, la importancia de las actividades para el aseguramiento de la calidad, y por otro lado, la necesidad de mejorar la coordinación de estas actividades para garantizar que la obtención productos con la calidad requerida por el cliente, convirtiéndose en actividades cada vez más comunes y vitales en el desarrollo de proyectos de software (Suali, Fauzi, Sobri, \& Nasir, 2017).

Esta necesidad se hace crítica en micro empresas, en las que una persona desarrolla más de un rol que conlleva la realización de múltiples actividades, y la mayoría de las veces no se cuenta con el conocimiento de expertos para la implementación de este tipo de actividades (Muñoz, M., Gasca G. \& Valtierra, C., 2014).

Como una solución a esta situación, este artículo presenta una herramienta cuyo objetivo es dar soporte a la implementación de las actividades para el aseguramiento de la calidad, con el fin de guiar y facilitar la implementación de éstas actividades en las empresas. Esta herramienta pretende mejorar tanto la comunicación, como el seguimiento y control en actividades relacionadas con la gestión de la calidad en proyectos de desarrollo de software, involucrando a los gestores de proyectos y los miembros del equipo.

Después de la introducción, este artículo está estructurado como sigue: en la sección 2 se presentan los trabajos relacionados; en la sección 3 se presenta la herramienta desarrollada; en la sección 4 se muestra el estudio de caso realizado; en la sección 5 se analizan los resultados y en la sección 6 se presentan los conclusiones. 


\section{Trabajos relacionados}

En (Heinemann, Hummel, \& Steidl, 2014), se propone una herramienta para realizar análisis de calidad por medio de pruebas dinámicas de integración de código utilizando un control de versiones, mediante la ejecución automática de una serie de pruebas por cada commit ejecutado.

En (Jain \& Gupta, 2011), se propone una herramienta para generar conjuntos de pruebas que satisfagan criterios de salida particulares, a partir del conjunto de pruebas se identifican debilidades y fortalezas de los conjuntos de pruebas.

En (Lin, He, Zhang, \& Song, 2015), se propone un marco de trabajo enfocado en la creación de un entorno que contenga un conjunto de herramientas por medio de un plan en el que se detalla las métricas y requisitos relevantes.

En (Mathrani, 2014), se propone un marco de trabajo enfocado al análisis dinámico de sistemas distribuidos, en donde los equipos de desarrollo realizan pruebas dinámicas al código para identificar y para resolver errores de código, facilitando la trazabilidad entre piezas de software y cambios realizados.

En (Öztürk, CİL, \& Zengin, 2015), se propone un marco de trabajo para facilitar el proceso de selección de herramientas para la ejecución de pruebas de código mediante una ontología de errores y herramientas ad-hoc para resolver determinados errores, proporcionado un conjunto base de herramientas para análisis dinámico de código.

En (Zaytsev \& Morrison, 2013), se propone un marco de trabajo para la integración continua del código mediante el control de versiones y un software para pruebas de integración automática de código.

$\mathrm{Al}$ analizar los trabajos relacionados, se observa, que la mayoría de las herramientas están enfocadas en la ejecución de pruebas dinámicas para resolver problemas de código, así como en la integración continua de los componentes de software. Sin embargo, controlar la calidad a partir del código potencializa el costo de corregir los errores detectados en esta etapa. Además, para que éstas herramientas se utilicen de forma adecuada, debe haber un previo entendimiento de qué se tiene artefactos se tienen que controlar a lo largo del desarrollo de software, así como los criterios y métricas que se van a utilizar. Este es un problema muy común que se identifica en las organizaciones y que es el enfoque de la herramienta propuesta en este artículo.

\section{Herramienta para la Gestión del Aseguramiento de la Calidad}

\subsection{Antecedentes}

Para el desarrollo de esta herramienta, se realizó en primer lugar una revisión sistemática (Muñoz, Mejía \& Ibarra, 2018) mediante la cual se obtuvieron los siguientes hallazgos:

a. Identificación de las principales actividades llevadas a cabo para el aseguramiento de la calidad: se identificaron 3 prácticas principales llevadas a cabo para el aseguramiento de la calidad de software: (1) identificar elementos relevantes para la calidad; (2)identificar y asignar métricas que tengan mayor impacto en la calidad de la organización; y (3) identificar y asignar criterios para 
la aceptación del producto. Estas actividades son establecidas por el Estándar IEEE 730 (McGrath et al. 2014), que es un estándar para el aseguramiento de la calidad, el cual establece los requisitos para iniciar, planificar, controlar y ejecutar el aseguramiento de la calidad dentro de un proyecto de desarrollo de software, y son mantenidas en modelos y estándares de calidad como CMMIDev v1.3, ISO 15504, MoProsoft, PMBOK.

Como parte del análisis a los resultados se identifico la falta de actividades importantes para realizar de manera correcta el aseguramiento de la calidad: (1) verificar la configuración del aseguramiento de la calidad y (2) realizar seguimiento y control de la calidad.

b. Identificación de un conjunto de herramientas para la gestión de la calidad: se identificó que además de herramientas, han sido desarrollados modelos, metamodelos y metodologías. La mayoría de estas herramientas se enfocan en la fase de desarrollo enfocado en el análisis estático y dinámico de código, la búsqueda, selección y ejecución de pruebas automáticas de software.

De los resultados mostrados anteriormente, se concluyó que las herramientas existentes no cubren todas las actividades principales para garantizar el aseguramiento de la calidad, debido a que éstas únicamente se enfocan en la fase de código y unas pocas en la verificación de los requisitos. Esta situación refleja el estado actual de la implementación de las actividades de aseguramiento de la calidad en las organizaciones, donde no existe un entendimiento común entre los gestores o líderes de proyecto, quienes realizan los planes para el aseguramiento de la calidad, y el equipo de trabajo quienes son los encargados de ir llevando a cabo las actividades relacionadas con el aseguramiento de calidad a través de las distintas etapas del desarrollo del software.

Por lo tanto, resalta la necesidad del desarrollo de una herramienta que sirva como plataforma de comunicación para la interacción de diferentes roles, en la que se tenga identificado en todo momento: (1) él o los artefactos de software se deben analizar; (2) los roles intervienen en la revisión y (3) las métricas establecidas, de tal manera que, se logre un entendimiento común entre los diferentes roles, de cómo sus actividades y productos de trabajo, suman para lograr la calidad del producto y para la toma de decisiones oportuna ante alguna desviación de los objetivos de calidad establecidos desde la planificación del proyecto.

\subsection{Desarrollo de la herramienta}

Tomando en cuanta los hallazgos de la revisión de literatura se identifica la necesidad de una herramienta que sirva como plataforma de comunicación y control para que las actividades de aseguramiento de calidad puedas ser llevadas a cabo durante el desarrollo de un proyecto de manera oportuna.

La herramienta desarrollada se estructura utilizando elementos del proceso, como a continuación se lista:

1. Fases: las fase representa cada una de las etapas del aseguramiento de la calidad, cada fase contiene una fecha límite, un conjunto de actividades y uno o más productos de trabajo que resultan de la ejecución de las actividades. 
2. Actividad: esta formada por un conjunto de tareas a realizar para la generación de una o más salidas, y que servirán de apoyo para dar por concluida la fase.

3. Tarea: acción específica que el equipo de trabajo debe realizar para producir las salidas y/o productos de trabajo requeridas por la actividad.

4. Productos de trabajo: elemento generado a través del ciclo de vida del desarrollo del proyecto, este puede ser: documentos, diagramas, código ejecutable, script, etc.

5. Salidas: elemento temporal que ayuda en el aseguramiento de la calidad de un producto o bien, proporciona información del estado actual de la calidad del producto de trabajo.

6. Roles: Define un conjunto de responsabilidades y habilidades específicas relacionadas con el aseguramiento de la calidad que deben ser llevadas a cabo dentro del proyecto de desarrollo de software por un miembro del equipo.

La Tabla 1 muestra la definición de los elementos de la herramienta propuesta, es importante resaltar que en la fase de inicio son configuradas las fases que apliquen al proyecto de acuerdo al ciclo de desarrollo de software utilizado.

\begin{tabular}{|c|c|c|c|c|}
\hline Fase & Descripción & Actividades & Producto de Trabajo & Roles \\
\hline Inicio & $\begin{array}{l}\text { Fase diseñada para } \\
\text { que el líder de proyecto } \\
\text { configure la herramienta, } \\
\text { seleccionado las fases, } \\
\text { productos, criterios y } \\
\text { métricas considerados } \\
\text { para el control de la } \\
\text { calidad. }\end{array}$ & $\begin{array}{l}\text { 1) Configurar la } \\
\text { herramienta respecto } \\
\text { al plan de calidad, plan } \\
\text { de Proyecto } \\
\text { 2) Asignar métricas de } \\
\text { calidad y criterios } \\
\text { de aceptación a los } \\
\text { productos de trabajo } \\
\text { agregados } \\
\text { 3) Verificar la aceptación } \\
\text { de la configuración de } \\
\text { la herramienta }\end{array}$ & $\begin{array}{l}\text { - Configuración de las } \\
\text { fases de desarrollo } \\
\text { - Configuración de } \\
\text { productos relevantes } \\
\text { - Criterios de aceptación } \\
\text { asignados a los } \\
\text { productos relevantes } \\
\text { - Métricas de calidad } \\
\text { asignadas a los } \\
\text { productos relevantes } \\
\text { - Configuración } \\
\text { aprobada }\end{array}$ & $\begin{array}{l}\text { Líder de } \\
\text { Proyecto } \\
\text { Responsable de } \\
\text { calidad }\end{array}$ \\
\hline Análisis & $\begin{array}{l}\text { Fase diseñada para } \\
\text { que el analista realice } \\
\text { las verificaciones } \\
\text { correspondientes y } \\
\text { el gerente de calidad } \\
\text { evalúe los resultados de } \\
\text { medición obtenidos }\end{array}$ & $\begin{array}{l}\text { 1) Evaluar la } \\
\text { aceptabilidad del } \\
\text { documento de } \\
\text { requisitos } \\
\text { 2) Analizar los resultados } \\
\text { de medición al } \\
\text { documento de } \\
\text { requisitos } \\
\end{array}$ & $\begin{array}{l}\text { - Documento de } \\
\text { requisitos verificado } \\
\text { - Documento de } \\
\text { requisitos validado } \\
\text { - No conformidades } \\
\text { respecto a los } \\
\text { resultados de medición }\end{array}$ & $\begin{array}{l}\text { Analista } \\
\text { Responsable de } \\
\text { calidad }\end{array}$ \\
\hline $\begin{array}{l}\text { Evaluación } \\
\text { de Riesgos }\end{array}$ & $\begin{array}{l}\text { Fase diseñada para que el } \\
\text { líder de proyecto verifique } \\
\text { el cumplimiento de los } \\
\text { criterios de aceptación } \\
\text { y el gerente de calidad } \\
\text { evalúe los resultados de } \\
\text { medición. }\end{array}$ & $\begin{array}{l}\text { 1) Evaluar la } \\
\text { aceptabilidad del } \\
\text { documento de riesgos } \\
\text { 2) Analizar los resultados } \\
\text { de medición al } \\
\text { documento de riesgos }\end{array}$ & $\begin{array}{l}\text { - Documento de gestión } \\
\text { de riesgos verificado } \\
\text { - No conformidades } \\
\text { respecto a los } \\
\text { resultados de medición }\end{array}$ & $\begin{array}{l}\text { Líder de } \\
\text { Proyecto } \\
\text { Responsable de } \\
\text { calidad }\end{array}$ \\
\hline
\end{tabular}




\begin{tabular}{|c|c|c|c|c|}
\hline Fase & Descripción & Actividades & Producto de Trabajo & Roles \\
\hline Diseño & $\begin{array}{l}\text { Fase diseñada para que el } \\
\text { arquitecto de software o } \\
\text { el responsable del diseño, } \\
\text { evalúé el cumplimiento de } \\
\text { los criterios de aceptación } \\
\text { registrados para el diseño } \\
\text { del sistema. }\end{array}$ & $\begin{array}{l}\text { 1) Evaluar la } \\
\text { conformidad del } \\
\text { diseño } \\
\text { 2) Evaluar la } \\
\text { aceptabilidad del } \\
\text { diseño del sistema } \\
\text { 3) Analizar los resultados } \\
\text { de medición al diseño } \\
\text { del sistema }\end{array}$ & $\begin{array}{l}\text { - Diagrama del sistema } \\
\text { verificado } \\
\text { - Diseño del sistema } \\
\text { verificado } \\
\text { - Diseño del sistema } \\
\text { validado } \\
\text { - No conformidades } \\
\text { respecto a resultados } \\
\text { esperados }\end{array}$ & $\begin{array}{l}\text { Arquitecto } \\
\text { de software/ } \\
\text { responsable de } \\
\text { diseño } \\
\text { Responsable de } \\
\text { calidad }\end{array}$ \\
\hline Desarrollo & $\begin{array}{l}\text { Fase diseñada para que } \\
\text { el desarrollador verifique } \\
\text { el cumplimiento de los } \\
\text { criterios de aceptación } \\
\text { establecidos para el } \\
\text { código fuente y el gerente } \\
\text { de calidad evalúe los } \\
\text { resultados de medición } \\
\text { obtenidos. }\end{array}$ & $\begin{array}{l}\text { 1) Evaluar conformidad } \\
\text { del código fuente } \\
\text { 2) Evaluar aceptabilidad } \\
\text { del código fuente } \\
\text { 3) Analizar los resultados } \\
\text { del código fuente }\end{array}$ & $\begin{array}{l}\text { - Diagramas del sistema } \\
\text { verificados contra } \\
\text { requisitos } \\
\text { - Diagramas del sistema } \\
\text { verificados contra el } \\
\text { diseño } \\
\text { - Código fuente } \\
\text { verificado } \\
\text { - No conformidades } \\
\text { respecto a los } \\
\text { resultados esperados }\end{array}$ & $\begin{array}{l}\text { Desarrollador } \\
\text { Responsable de } \\
\text { calidad }\end{array}$ \\
\hline Pruebas & $\begin{array}{l}\text { Fase diseñada para } \\
\text { que el téster verifique } \\
\text { el cumplimiento de los } \\
\text { criterios de aceptación, } \\
\text { de igual manera que } \\
\text { el gerente de calidad } \\
\text { evalúe los resultados de } \\
\text { medición obtenidos. }\end{array}$ & $\begin{array}{l}\text { 1) Evaluar la } \\
\text { conformidad del } \\
\text { plan de pruebas y/o } \\
\text { el procedimiento de } \\
\text { pruebas } \\
\text { 2) Evaluar la } \\
\text { aceptabilidad del } \\
\text { plan de pruebas y los } \\
\text { resultados de pruebas } \\
\text { 3) Análisis de los } \\
\text { resultados de medición } \\
\text { del plan de pruebas } \\
\text { y los resultados de la } \\
\text { pruebas }\end{array}$ & $\begin{array}{l}\text { - Plan de pruebas o } \\
\text { procedimientos de } \\
\text { pruebas verificados } \\
\text { contra los requisitos } \\
\text { - Plan de pruebas y } \\
\text { resultados de pruebas } \\
\text { verificados } \\
\text { - Resultados de pruebas } \\
\text { validados } \\
\text { - No conformidades } \\
\text { respecto a los } \\
\text { resultados esperados }\end{array}$ & $\begin{array}{l}\text { Téster } \\
\text { Responsable de } \\
\text { calidad }\end{array}$ \\
\hline Liberación & $\begin{array}{l}\text { Fase diseñada para que el } \\
\text { líder de proyecto verifique } \\
\text { el cumplimiento de los } \\
\text { criterios de aceptación } \\
\text { y el gerente de calidad } \\
\text { evalúe los resultados de } \\
\text { medición obtenidos del } \\
\text { procedimiento de entrega }\end{array}$ & $\begin{array}{l}\text { 1) Evaluar aceptabilidad } \\
\text { del procedimiento de } \\
\text { entrega } \\
\text { 2) Análisis de los } \\
\text { resultados de medición } \\
\text { del procedimiento de } \\
\text { entrega }\end{array}$ & $\begin{array}{l}\text { - Procedimiento de } \\
\text { entrega verificado } \\
\text { - procedimiento de } \\
\text { entrega validado } \\
\text { - No conformidades } \\
\text { respecto a los } \\
\text { resultados esperados }\end{array}$ & $\begin{array}{l}\text { Líder de } \\
\text { Proyecto } \\
\text { Responsable de } \\
\text { calidad }\end{array}$ \\
\hline Cierre & $\begin{array}{l}\text { Fase diseñada para que el } \\
\text { líder de proyecto confirme } \\
\text { el cumplimiento de los } \\
\text { criterios de aceptación y } \\
\text { de igual manera el gerente } \\
\text { de calidad evalúe los } \\
\text { resultados de medición } \\
\text { obtenidos. }\end{array}$ & $\begin{array}{l}\text { 1) Evaluar aceptabilidad } \\
\text { del procedimiento de } \\
\text { entrega } \\
\text { 2) Mediciones de calidad } \\
\text { al procedimiento de } \\
\text { entrega }\end{array}$ & $\begin{array}{l}\text { - Documento de cierre de } \\
\text { proyecto verificado }\end{array}$ & $\begin{array}{l}\text { Líder de } \\
\text { proyecto }\end{array}$ \\
\hline
\end{tabular}

Tabla 1 - Definición de las fases de la herramienta 
Como se observa en la Tabla cada fase ha sido diseñada para disminuir la brecha de entendimiento en cuanto al aseguramiento de calidad de las personas involucradas en el desarrollo de un proyecto, como a continuación se describe:

- Mejorar el común entendimiento referente a la calidad de producto debido a que la herramienta está diseñada para todos miembros de un equipo de desarrollo de software tengan un común entendimiento referente a la visualización de la calidad por parte del responsable de calidad y el líder del proyecto.

- Permite la configuración de las fases en las que se vallevar a cabo el aseguramiento de la calidad con base en el ciclo de desarrollo de software utilizado.

- Mejora el conocimiento de cómo se llevará a cabo el aseguramiento de la calidad a lo largo de proyecto, ya que permite la selección de artefactos de proyecto por fase que debe ser medidos y los criterios que deben cubrir.

- Facilita a los miembros del equipo a realizar actividades de aseguramiento de calidad dependiendo de su rol y fase del ciclo de desarrollo de software.

- En cada fase se implementan las 3 actividades principales para el aseguramiento de la calidad. Además, en la primera fase de implementa la actividad adicional de verificar la configuración, la cual asegura que todos están de acuerdo en las actividades de aseguramiento de calidad a llevarse a cabo en el proyecto. Finalmente, con la ejecución de la herramienta se está cumpliendo con la segunda actividad adicional referente a realizar seguimiento y control de la calidad.

\subsection{Ejemplo de interfaz de la herramienta}

El diseño completo de la herramienta propuesta, así como algunas de sus pantallas, se encuentra publicado en (Ibarra \& Muñoz, 2018). En esta sección se incluye a manera de ejemplo un conjunto de pantallas significativas para mostrar los elementos de la herramienta.

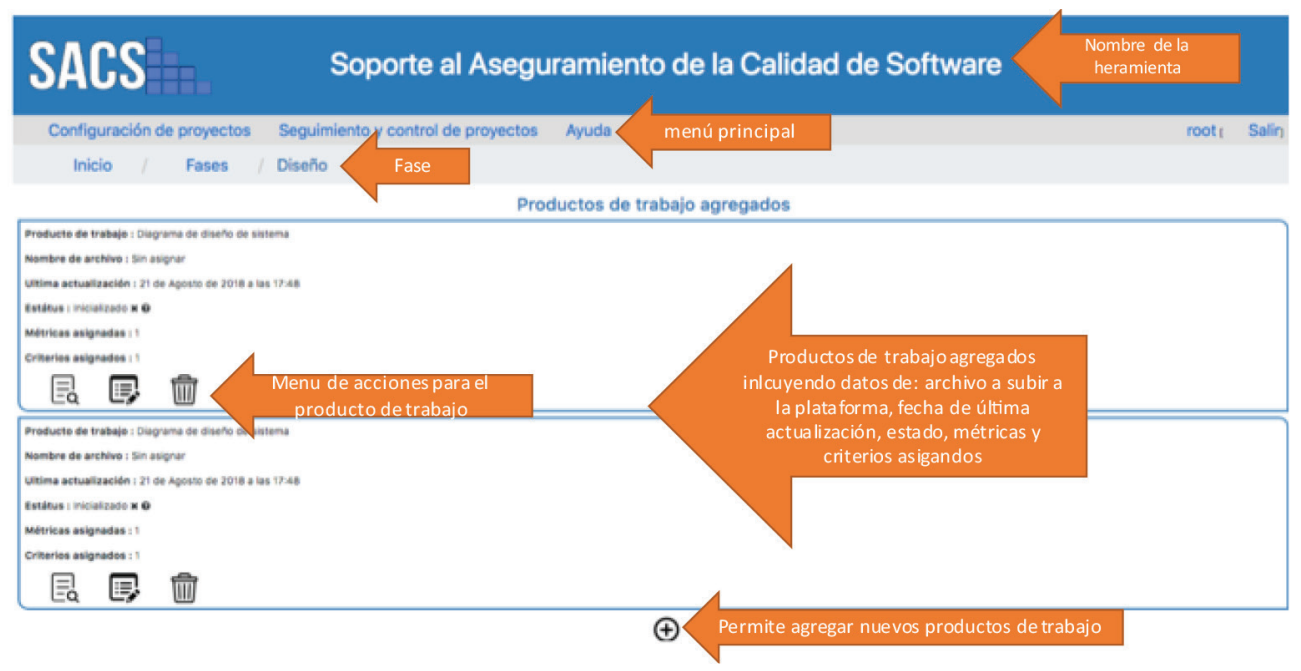

Figura 1 - Pantalla de la herramienta para agregar productos de trabajo de la fase de Diseño 
La Figura 1, muestra la pantalla de la fase de diseño, en la que se tienen agregados dos productos de trabajo a ser revisados como parte del aseguramiento de la calidad.

La Figura 2 muestra la pantalla de los criterios y métricas asignadas para el producto de trabajo de la fase de diseño: Diagrama de diseño del sistema.

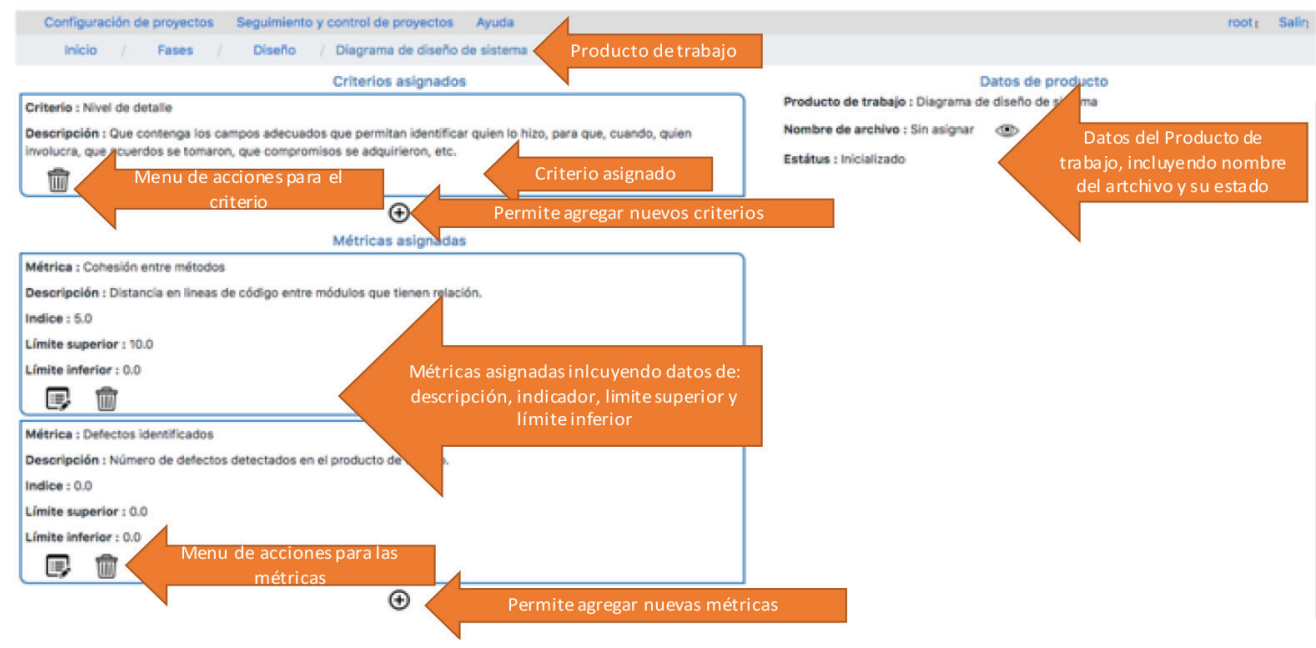

Figura 2 - Pantalla de gestión de criterios y métricas para un producto de trabajo de la fase de Diseño

La Figura 3 muestra la pantalla para la revisión de los resultados de medición.

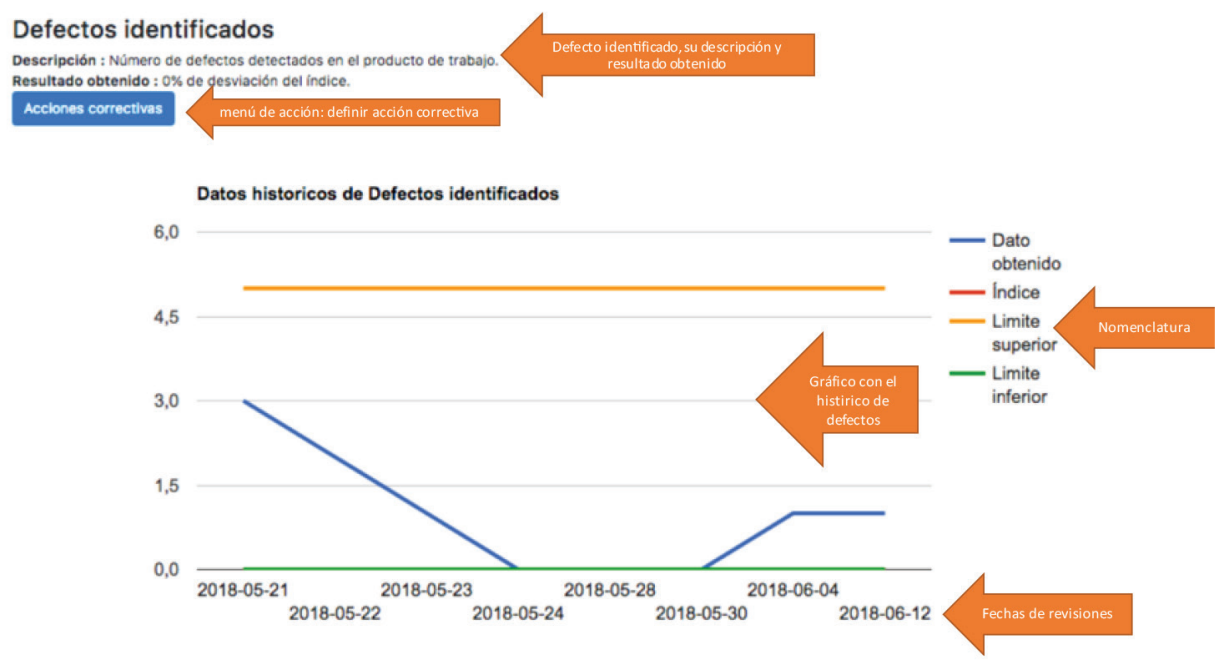

Figura 3 - Pantalla en la que se revisan los resultados de medición [adaptado de (Ibarra \& Muñoz, 2018)] 
La Figura 4 muestra la pantalla para establecimiento de acciones correctivas según sea necesario.

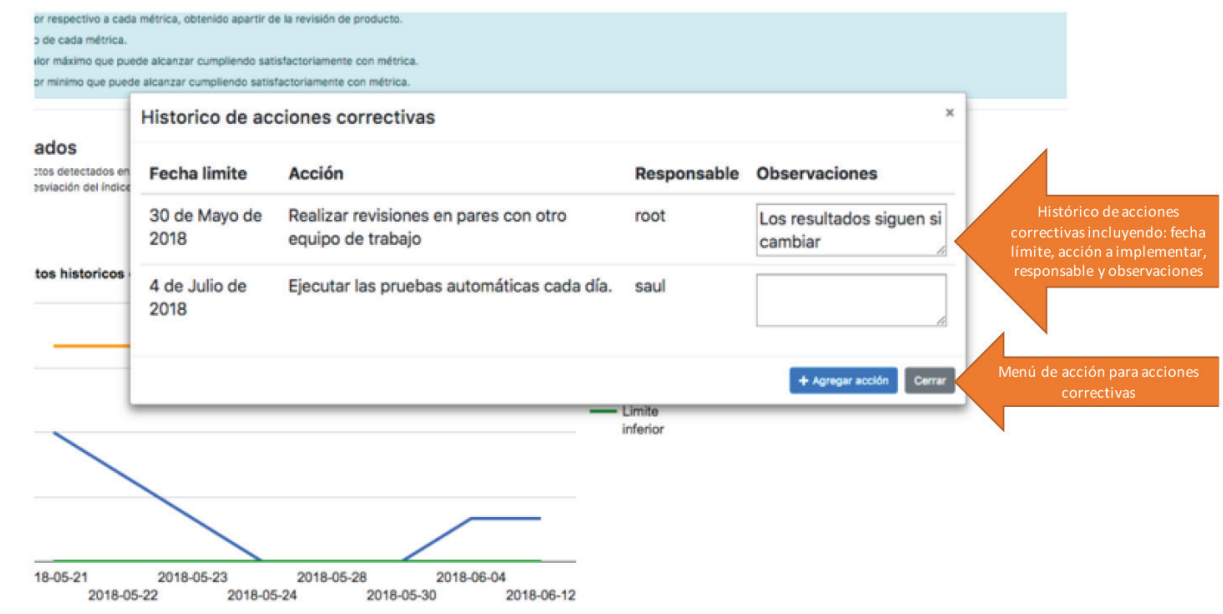

Figura 4 - Pantalla en la que se establecen acciones correctivas con base en resultados de medición [adaptado de (Ibarra \& Muñoz, 2018)]

\section{Estudio de Caso}

Para validar la herramienta se realizó un estudio de caso en el que participaron 4 empresas, de las cuales 2 son centros de desarrollo de software de universidades, un centro de investigación y una microempresa de desarrollo de software.

El objetivo del estudio fue evaluar el nivel en el que las prácticas integradas como parte de herramienta eran percibidas el conjunto de empresas de desarrollo de software para apoyarlas en la realización de las actividades de aseguramiento de software que éstas llevan a cabo.

Las empresas de desarrollo de software participantes se describen a continuación:

1. Empresa 1: Es un Centro de Desarrollo de Software dentro de una universidad, que se dedica a desarrollar software para clientes internos y externos a la institución, certificad en el estándar ISO/IEC 29110. De este centro de desarrollo de software se encuestaron 4 personas con los siguientes roles: desarrollador, téster, diseñador y gestor.

2. Empresa 2: Es un Centro de Desarrollo de Software dentro de una universidad, que se dedica a desarrollar software para clientes internos y externos a la institución, certificado en el estándar ISO/IEC 29110. De este centro de desarrollo de software se encuestó a 1 persona con el rol de gestor.

3. Empresa 3: Es una microempresa de desarrollo de software embebido, certificada en el estándar ISO/IEC 29110. De esta empresa se encuestó a e persona con el rol de desarrollador, téster, diseñador y analista. 
4. Empresa 4: Laboratorio de un centro de investigación en el que se integran equipos de profesionales para trabajar en proyectos específicos. De este centro de laboratorio se encuestaron 4 personas con los siguientes roles: desarrolladores, analistas, téster, arquitectos y gerentes de calidad.

Es importante resaltar que de las 10 personas participantes en el estudio de caso, no todas tienen la misma experiencia, 1 persona tiene menos de un año de experiencia; 5 personas tienen de uno a tres años de experiencia y 4 personas tienen más de cinco años de experiencia en la realización de actividades relacionadas con la calidad y el aseguramiento de la calidad.

Las preguntas de investigación definida:

1. ¿La herramienta facilita la gestión de la calidad en el desarrollo de software?

2. ¿Las prácticas contenidas en la herramienta permiten implementar el aseguramiento de la calidad del producto?.

3. ¿Se mejora la identificación de defectos en los productos de software obtenidos?.

Para la recogida de datos se diseñó un cuestionario en google forms, dividido en dos secciones; la primera sección enfocada en la percepción de los elementos de aseguramiento de calidad y la segunda sección en la precepción de la herramienta. El cuestionario competo se lista a continuación:

a. Sección enfocada en los elementos de aseguramiento de la calidad incluidos como parte de la herramienta:

1. ¿Considera que las fases disponibles son las adecuadas para la cobertura toral de la metodología de desarrollo que realiza en su trabajo?

2. ¿Considera que los tipos de producto de trabajo disponibles son suficientes para llevar acabo en aseguramiento de calidad básico?

3. ¿Considera que las actividades de aseguramiento de calidad que realiza se pudieron ajustar a la configuración de la herramienta?

4. ¿Considera que con las actividades realizadas se obtendrá una mejora en la prevención de defectos?

b. Sección enfocada en la herramienta:

- Facilidad de entendimiento

1. ¿Se reduce el esfuerzo para realizar las acciones en pocos pasos?

2. ¿El estado de la calidad se transmite en forma clara y concisa?

3. ¿La herramienta permite fácilmente al usuario identificar qué acciones debe llevar a cabo?

- Facilidad de uso

1. ¿Los usuarios realizan sus tareas en el menor tiempo posible?

2. ¿Las tareas están diseñadas para realizarse de la forma más rápida e intuitiva posible?

- Satisfacción en uso

1. ¿Los resultados que obtendrías tras la interacción con la aplicación web son los deseados?

2. ¿La interfaz es amigable al usuario?

El proceso de recogida de datos consistió en 5 pasos listados a continuación: 
1. Realizar una reunión con cada una de las empresas participantes, durante la cuál se muestra la herramienta, describiendo de manera breve su funcionamiento.

2. Una vez finalizada la presentación, se proporciona acceso a la herramienta.

3. Se permite a los participantes interactuar con la herramienta.

4. Se proporciona el link del cuestionario y se invita a los participantes a responder la encuesta.

5. Se analizan los resultados.

Los resultados obtenidos de la ejecución del estudio de caso se analizan en la siguiente sección.

\section{Análisis de resultados.}

En esta sección se mostrarán los resultados mediante la respuesta de cada una de las preguntas definidas en el estudio de caso.

Pregunta 1. ¿La herramienta facilita la gestión de la calidad en el desarrollo de software?

Para responder la pregunta 1 se analizaron las respuestas de la segunda sección del cuestionario enfocada en la herramienta. La Figura 4 muestra un resumen de los datos.

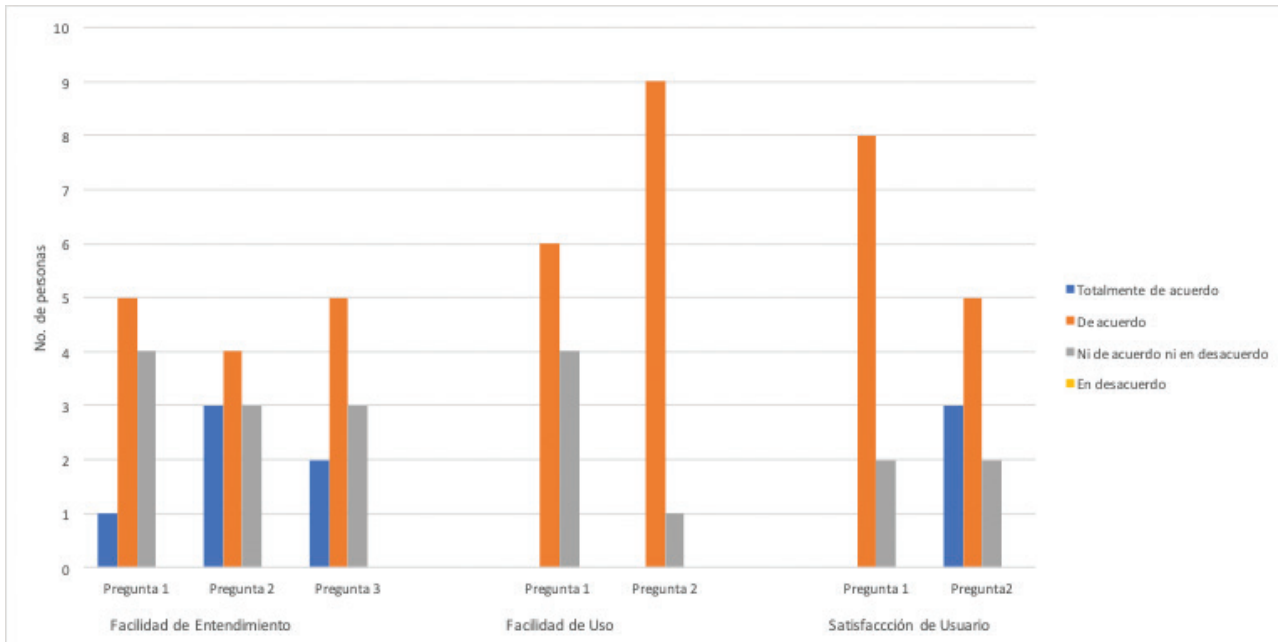

Figura 5- Gráfico de respuestas de preguntas relacionadas con la percepción de facilidad del aseguramiento de la calidad mediante el uso de la herramienta

a. Bloque de preguntas relacionadas con la facilidad de entendimiento:

- En cuanto a la pregunta 1 relacionada con la reducción del esfuerzo para realizar acciones en pocos pasos el 50\% de los encuestados está de acuerdo y $10 \%$ de los encuestados está totalmente de acuerdo, mientras que 40\% de los encuestados tiene una opinión neutral, por lo que se considera que 
la herramienta puede apoyar en la reducción de esfuerzo para la realización de las actividades, aunque es importante identificar los puntos de mejora de la misma.

- En cuanto a la pregunta 2 relacionada con la transmisión del estado de la calidad 30\% de los encuestados están totalmente de acuerdo; 40\% de los encuestados está de acuerdo; y 30\% de los encuestados tienen una opinión neutra, por lo que se considera que la herramienta apoya en la comunicación y transmisión del estado de la calidad del producto en sus diferentes fases.

- En cuanto a la pregunta 3 relacionada con facilitar la identificación de las acciones que se tienen que llevar a cabo por usuario (rol), 10\% de los encuestados está totalmente de acuerdo, 50\% de los encuestados están de acuerdo y 30\% de los encuestados tiene una opinión neutra, por lo que se considera que la herramienta es percibida de apoyo para que los miembros de un equipo tengan conocimiento de sus actividades referente a la calidad a través de las distintas fases del desarrollo de software.

b. Bloque de preguntas relacionadas con la facilidad de uso:

- En cuanto a la pregunta 1 relacionada con la correcta realización de las tareas en menor tiempo posible, 60\% de los encuestados están de acuerdo y 40\% de los encuestados tienen una opinión neutra, por lo que se considera que la herramienta es percibida como fácil de usar por la mayoría de los encuestados. Aunque deben detectarse oportunidades de mejora de los 40\% encuestados que tienen opinión neutra

- En cuanto a la pregunta 2 relacionada con el diseño de la herramienta para realizar las tareas de forma más rápida e intuitiva, 90\% de los encuestados están de acuerdo y sólo el 10\% tiene una opinión neutra, por lo que se considera que las tareas están diseñadas para realizarse de una forma más rápida e intuitiva.

c. Bloque de preguntas relacionadas con la satisfacción:

- En cuanto a la pregunta 1 relacionada con los resultados a la percepción de si se obtendrían los resultados deseados con la aplicación web, 80\% de los encuestados están de acuerdo y 20\% de los encuestados tienen una opinión neutra, por lo que se considera que con la implementación de la herramienta se obtendrá una mejora en la gestión de las actividades de calidad de los productos de trabajo.

- En cuanto a la pregunta 2 relacionada con los resultados de si la interfaz es percibida como amigable, 30\% de los encuestados están totalmente de acuerdo; 50\% de los encuestados están de acuerdo y el 20\% de los encuestados tienen una opinión neutra, por lo que se considera que la interfaz de la herramienta es amigable.

Pregunta 2. ¿Las prácticas contenidas en la herramienta permiten implementar el aseguramiento de la calidad del producto?.

Para responder la pregunta 2 se analizaron las respuestas de las preguntas 1, 2 y 3 del cuestionario. La Figura 5 muestra un resumen de los datos.

En cuanto a la pregunta 1 del cuestionario, relacionada con la percepción de si las fases incluidas en la herramienta permiten cubrir las fases de la metodología usada en la 
empresa, como se observa en la figura, el 80\% están de acuerdo y el 20\% totalmente de acuerdo, por lo que se considera que la herramienta cubre las fases de desarrollo de software y pueden ser adecuadas a su forma de trabajo.

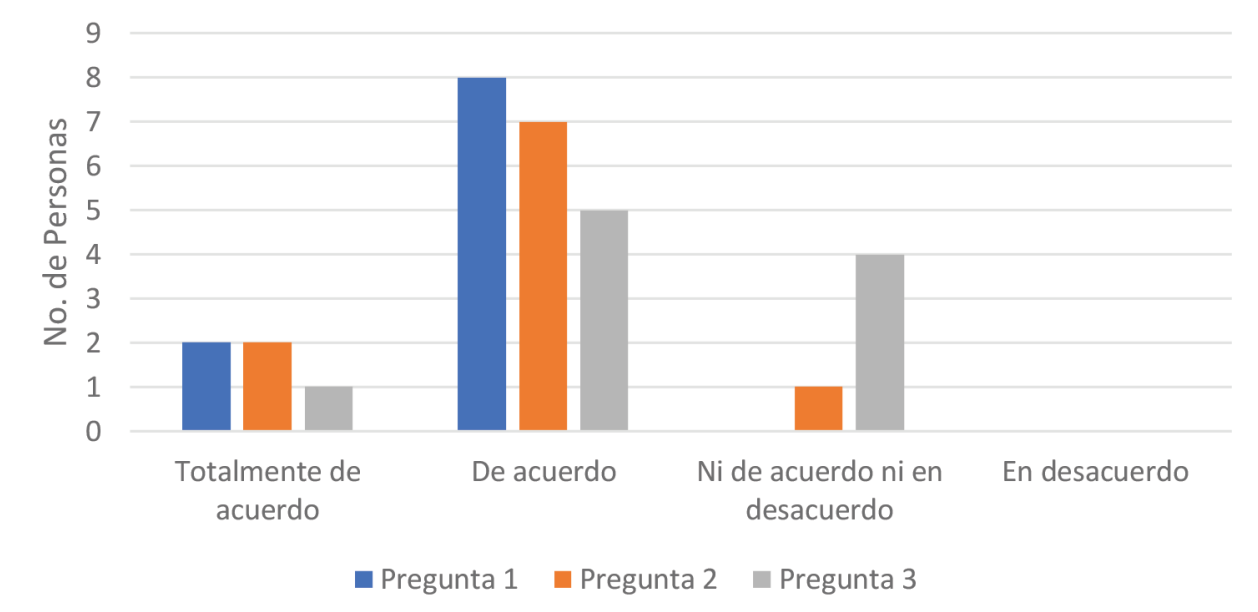

Figura 6 - Gráfico de respuestas de preguntas relacionadas con la percepción de facilidad en el aseguramiento de la calidad

En cuanto a la pregunta 2 del cuestionario, relacionada con si los productos de trabajo considerados en la herramienta son suficientes para una gestión básica del aseguramiento de la calidad, como se observa en la figura el 20\% está totalmente de acuerdo y el 70\% está de acuerdo, y únicamente el 10\% tiene una percepción neutra, por lo que se considera que la herramienta contiene los productos de trabajo que permiten llevar a cabo una gestión básica del aseguramiento de la calidad.

Finalmente, en cuanto a la pregunta 3, relacionada con si las actividades que normalmente llevan a cabo para el aseguramiento de la calidad pueden ser configuradas utilizando la herramienta, como se observa en la figura aquí hay un cambio de percepción ya que el 10\% están totalmente de acuerdo y el 50\% está de acuerdo. Sin embargo el 40\% tiene una percepción neutra. Al ser la mayoría la que considera que pueden ser configuradas sus actividades, se considera que la herramienta apoya en la implementación de actividades para el aseguramiento de la calidad.

Pregunta 3. ¿Se mejora la identificación de defectos en los productos de software obtenidos?.

Para responder la pregunta 3 se analizó la respuesta 4 de la primera sección del cuestionario, relacionada con la percepción de una mejora en la prevención de los defectos, el 40\% de los encuestados está totalmente de acuerdo; 40\% de los encuestados está de acuerdo y el 20\% tiene una opinión neutra, por lo que se concluye que la herramienta apoyará en la reducción de los defectos. 
Es importante resaltar que como limitante del caso de estudio únicamente se tuvo una sesión, sin embargo ninguna de las empresas utilizaron la herramienta en un desarrollo de software completo, aún cuando tuvieron acceso a la misma, únicamente exploraron su contenido, y pudieron revisar el ejemplo cargado en la misma.

\section{Conclusiones.}

El aseguramiento de la calidad es una actividad esencial en los proyectos de desarrollo, desafortunadamente aún cuando se establezcan planes de mejora, éstos no son transmitidos de manera adecuada al equipo de trabajo, lo que resulta en una falta de entendimiento en actividades a realizar y en cómo se va gestionando la calidad a través del ciclo de desarrollo de software. Esta situación e hace más crítica en PYMES y MiPYMES donde cada miembro del equipo ejecuta más de un rol.

Como una solución a esta situación, en este artículo se presenta una propuesta de herramienta software cuyo objetivo es brindar soporte a los equipos de desarrollo para la gestión de las actividades relacionadas con el aseguramiento de la calidad.

El estudio de caso realizado, en el que mostró la herramienta a un conjunto de 4 empresas, las cuales tuvieron acceso a la misma y pudieron explorarla y revisar el ejemplo precargado de la misma, demuestra que la herramienta es percibida cómo útil para soportar éstas actividades relacionada con el aseguramiento de la calidad, teniendo como principales características: (1) implementa las 3 actividades principales de aseguramiento de calidad; (2) es fácilmente configurable de acuerdo a las fases del ciclo de desarrollo de software utilizado en la organización; (3) facilita a los integrantes de un equipo el conocer los productos de trabajo a revisar y las fechas en las que debe realizarse; (4) habilita una plataforma de comunicación para obtener un entendimiento común entre el gestor o líder un proyecto y el equipo de trabajo relacionado con las actividades que deben llevarse a cabo para el aseguramiento de la calidad y (5) permite el análisis de los resultados y; el establecimiento y seguimiento de acciones correctivas.

Es importante mencionar que el estudio de caso fue realizado con una muestra pequeña de empresas, y con restricciones como la falta de uso de la herramienta durante todo un proyecto de desarrollo de software, y que se establece como trabajo futuro.

Finalmente, con base en los resultados obtenidos se concluye que con la realización de este tipo de herramientas puede ser disminuida la brecha entre gestores de proyecto y equipo de trabajo hacía una visión más clara de la gestión de la calidad, mediante la implementación de prácticas para el aseguramiento de la calidad.

\section{Agradecimientos}

Al alumno Saúl Alonso Ibarra Luévano quien ha desarrollado la herramienta presentada en este artículo como parte de su tesis para obtención del grado de Maestro en Ingeniería de Software ofrecida en el Centro de Investigación en Matemáticas. 


\section{Referencias}

Heinemann, L., Hummel, B., \& Steidl, D. (2014). Teamscale: software quality control in realtime. In: Companion Proceedings of the 36th International Conference on Software Engineering - ICSE 2014, (pp.592-595). New York, New York, USA: ACM Press. doi:10.1145/2591062.2591068.

Ibarra, S., \& Muñoz, M. (2018). Support tool for software quality assurance in software development. In: Proceedings of the 7 th, International Conference On Software Process Improvement (CIMPS), (pp.13-19). IEEE. doi: 10.1109/ CIMPS.2018.8625617.

Jain, R. S., \& Gupta, A. (2011). Refinement of the Test Bed Using Various Prioritization Techniques for Assuring Software-Quality (pp. 657-661). Berlin: Springer. doi:10.1007/978-3-642-25734-6_113.

Lin, L., He, J., Zhang, Y., \& Song, F. (2015). Quality Assurance through Rigorous Software Specification and Testing: A Case Study. Procedia Computer Science, 62, 257-265. doi: 10.1016/j.procs.2015.08.448.

Mathrani, A. (2014). Quality assurance strategy for distributed software development using managed test lab model. In: 2014 IEEE International Technology Management Conference (pp. 1-4). Doi: 10.1109/ITMC.2014.6918609.

Muñoz, M., Mejía, J., \& Ibarra, S. (2013). Herramientas y prácticas para el aseguramiento de la calidad del software: Una revisión sistemática. IEEEXplore.

Muñoz, M., Gasca, G., \& Valtierra, C. (2014). Caracterizando las necesidades de las pymes para implementar mejoras de procesos software: una comparativa entre la teoría y la realidad. Revista Ibérica de Sistemas y Tecnologías de Información, E1, $1-15$.

Öztürk, M. M., CİL, İ., \& Zengin, A. (2015). Development of a Multi-Agent Framework for Software Quality. ACM SIGSOFT Software Engineering Notes, 40(1), 1-10. Doi: 10.1145/2693208.2693234.

Rebolledo, R., (2017). Las 20 mejores empresas del mundo en el 2017. El Economista, [Online]. Available: https://www.eleconomista.com.mx/empresas/Las-20mejores-empresas-del-mundo-en-el-2017-20170628-0113.html.

Suali, A. J., Fauzi, S. M., Sobri, W. A. W. M., \& Nasir, M. H. N. M. (2017). Developers' coordination issues and its impact on software quality: A systematic review. In: 2017 3rd International Conference on Science in Information Technology (ICSITech), (pp. 659-663).

Zaytsev, Y. V., \& Morrison, A. (2013). Increasing quality and managing complexity in Neuroinformatics Software Development with Continuous Integration. Frontiers in Neuroinformatics, 6, 31. Doi: 10.3389/fninf.2012.00031. 